\title{
A Review on Biofeedback System For Obstructive Sleep Apnea In Geriatric Population
}

\author{
Monisha $\mathbf{R}^{1}$, Premkumar $\mathbf{R}^{2}$ \\ PG Scholar, Department of Biomedical Engineering, REC, Chennai ${ }^{1}$ \\ Associate Professor, Department of Biomedical Engineering, REC, Chennai ${ }^{2}$
}

\begin{abstract}
Sleep is essential for humans although its basic physiological function remains obscure. Humans suffer from various sleep disorders including dyssomnias such as insomnia, hypersomnia, narcolepsy, parasomnias, bruxism, circadian rhythm sleep disorders and sleep apnea. The major problem addressed in this review paper is Obstructive Sleep Apnea in geriatric population. Sleep Apnea is defined as hinderance of airflow during breathing where soft tissue muscle in throat relax and narrow the airway which cause blockage of upper airway tract (Obstructive sleep apnea). Microcontroller based sleep apnea monitor consists of a Respiration sensor for measuring and monitoring the breath condition, SPO2 and Pulse Sensor for monitoring the heart rate and blood oxygen saturation level and Digital Humidity and Temperature sensor. All the parameters are monitored by the microcontroller once the microcontroller detects any difficulties in breathing conditions it'll automatically turn on the supportive system for the patient. By using our proposed supportive system patients can able to breathe stably. In addition, the acquired parameters will be accessible to the practitioner through customised IoT platform which is inbuilt in system.
\end{abstract}

Keywords: Dyssomnia, Obstructive sleep Apnea, Geriatric population, Microcontroller and IoT

\section{INTRODUCTION}

Sleep apnea is significant sleep disorder which causes soft tissue muscle relaxation during breathing which narrow the airway tract, where breathing is cuttoff temporarily and obstruct air flow. During each halt there will be no movement in throat muscles. In the most common form, this follows loud snoring and there cause abrupt awakening causing gasping or choking. Main three types of sleep apnea include, Obstructive sleep apnea (OSA) the more common form that occurs when throat muscles relax (Fig. 1), Central sleep apnea which occurs when your brain doesn't send proper signals to the muscles that control breathing and Complex sleep apnea syndrome which occurs when someone has both obstructive sleep apnea and central sleep apnea. Whereas Obstructive Sleep Apnea (OSA) is the most common form. It affects males about twice as often as females. OSÁ has been diagnosed more in 55 and above age group [1,2]. Approximately 1 billion of the world's population of 7.3 billion people, between the ages of 30 and 69 years, are estimated to have the most common type of sleep-disordered breathing, obstructive sleep apnoea (OSA) [3] Prevalence surveys have estimated that about $4 \%$ of the middle-aged men and $2 \%$ of the middle-aged women are afflicted by OSA in developed countries. 1$2.1 \%$ in females) [4]. There are few existing methods to treat OSA such as Polysomnography and apnea monitor, each technique has unique pros and cons like Apnea monitor requires technician to assist the patients whereas the cost of polysomnography is high. In order to address the existing drawbacks, the proposed system will be incorporated with multiple features to monitor, oxygen saturation level, Abrupt awakening and Cessation of airflow. Pause in breathing is monitored using a respiration sensor and the corresponding therapeutic support will be provided when the breathing is paused. Accelerometer sensor is used to monitor the abrupt awakening of the patient and decrease in oxygen saturation levels are monitored continuously by the $\mathrm{SpO} 2$ sensor along with Heart rate calculation. The acquired parameters are encrypted and transmitted to practitioner via inbuilt IoT module. The objective of the review paper is to analyse existing methodology and its drawbacks. 

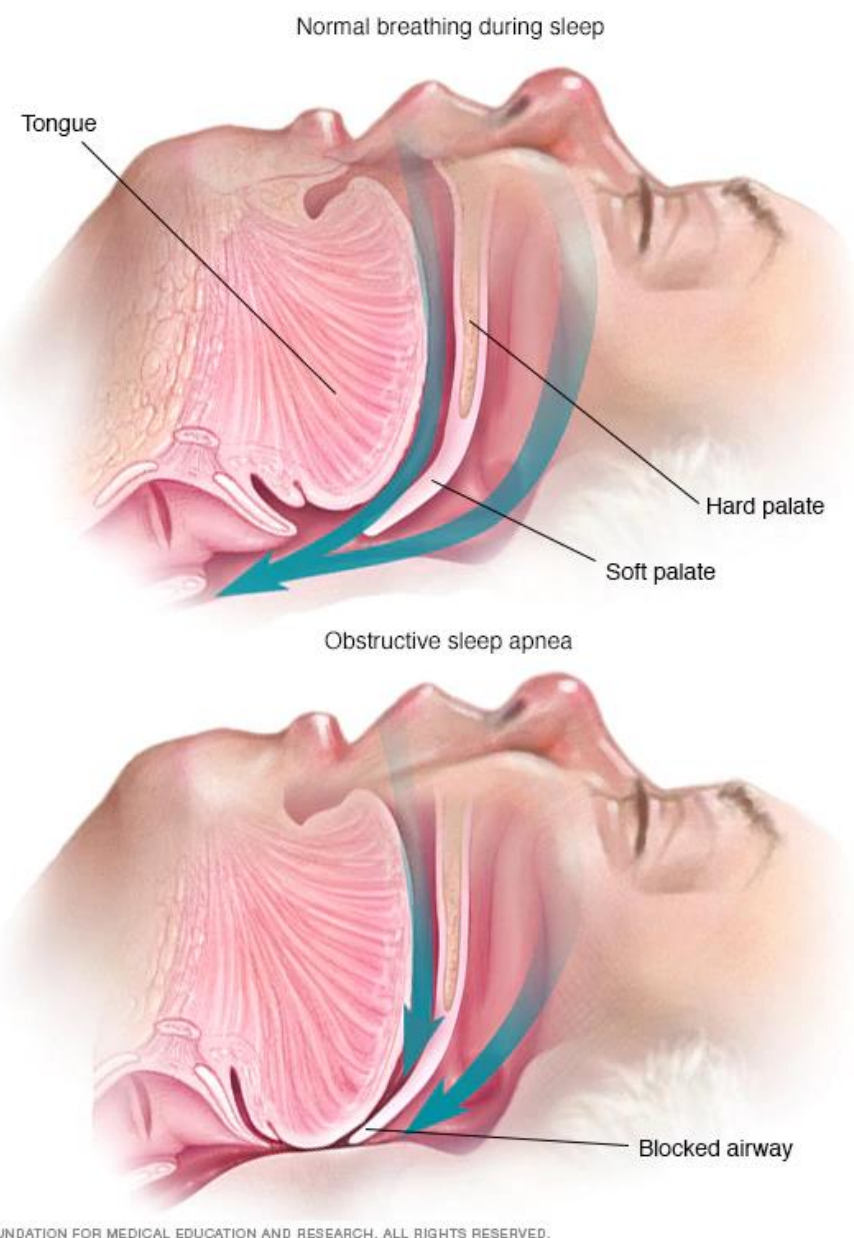

Fig. 1: Normal subject vs. Obstructive sleep apnea subject

(Courtesy: https://www.mayoclinic.org/diseases-conditions/obstructive-sleep-apnea/symptoms-causes/syc-20352090)

\section{REVIEW ON EXISTING METHODOLOGIES}

The existing method based on oxygen saturation, respiration sound and combined approaches will be discussed below with corresponding research details.

- Review on $\mathrm{SpO} 2$ sensor

Oximetry is a method utilized for estimating the oxygen immersion (SpO2) in the blood, ordinarily performed by placing $\mathrm{SpO} 2$ sensor at subject's extremities. Oliver and Flores-Mangas [5] introduced a gadget, comprising of an oximetry sensor that sends information to be inspected by Bluetooth to a cell phone. The OSA identifying calculation registers a periodogram of the mean-deducted oximetry sign and investigations the recurrence range from 0.015 to $0.04 \mathrm{~Hz}$ to distinguish power tops. Burgos et al. [6] constructed a gadget that sends the oximeter information by Bluetooth to a Personal Digital Assistant (PDA). An order measure, assessing five rotating choice trees and getting the larger part vote, was performed for each SpO2 signal piece. A synchronous discovery and treatment approach was introduced by Zhang et al. [7]. A heartbeat oximeter peruses the $\mathrm{SpO} 2$ level and sends the information, by Bluetooth, to a cell phone to be broke down by a calculation that looks for desaturation scenes. First it distinguishes a nearby least SpO2 point and the closest neighborhood most extreme $\mathrm{SpO} 2$ point. Measurable examination was performed on the time space and the force otherworldly thickness (PSD) was resolved, through an autoregressive model. A straight discriminant examination (LDA) was utilized as classifier. A similar gadget was utilized by Garde et al. [8] however the beat oximeter produces both SpO2 and photoplethysmography (PPG) signals. The calculation investigations these signs in both time and recurrence spaces. A remote methodology was utilized by Angius and Raffo [9], estimating the PPG signal from the SpO2 sensor and sending 


\section{International Journal of Advanced Research in Computer and Communication Engineering}

Vol. 9, Issue 12, December 2020

DOI 10.17148/IJARCCE.2020.91204

information to an observing station. The drawback of the existing study is that the data cannot be shared widely. But in proposed solution pulse oximeter sensor (MAX30100) will be integrated with IoT for wide range of access at low cost.

- Review on respiration sensor

During the occasion of air blockage, the breathing sounds of the subject can be utilized to identify apnea. This rule was utilized by Kaniusas et al. [10]. Breathing (wheezing, Snoring) sounds were recorded by a mouthpiece set close to the chest and the order of breathing occasions was performed. Versatile ranges and histogram examination were utilized to recognize OSA on the grouped occasions. Tracheal sounds were investigated by Nakano et al. [11] utilizing a packed sound spectrograph framework. A tracheal sound-respiratory unsettling influence file was proposed to assess OSA. An alternate methodology was introduced by Zhao et al. [12], where the amplifier was set over the patient's mouth to gather breathing sounds. A clustering algorithm was used to partition the snorer's first formant frequencies into two groups utilizing a customized edge. Cell phones were utilized by a few scientists to record sounds. Lu-Ping et al. [13] utilized Mel-recurrence cepstral coefficients and a clustering calculation on the recorded sound to identify apnea. With a similar objective Ren et al. [14] what's more, Han et al. [15] applied SVM as a classifier. Nandakumar et al. [16] utilized a cell phone as a functioning sonar framework. The telephone speaker communicated sound waves and their reflections were identified by the amplifier. A recurrence adjusted wave was produced and a calculation analyzed the reflections that showed up at various occasions, focusing on the reflection designs created from breathing to appraise the breathing sign. OSA was assessed utilizing a pinnacle recognition calculation. Based on this existing study, the add-on feature incorporated with respiration sensor. The automated process of supportive system will be initiated when patient faces trouble in breathing.

- Review on market available products

A five-channel gadget, Apnoescreen-I (CNS-Jaeger, Germany), was inspected by Golpe et al. [17] estimating the oronasal output with a thermistor, and oxygen immersion with finger heartbeat oximetry, to acquire the respiratory unsettling influence list. A wrist-mounted gadget, WristOx 3100 (Nonin, USA), was concentrated by Nigro et al. [18], utilizing a finger test to gauge the $\mathrm{SpO} 2$, computing the mean number of oxygen desaturations per hour, the measure of rate dip under the $\mathrm{SpO} 2$ mean worth furthermore, the level of amassed time with $\mathrm{SpO} 2$ lower than $90 \%$. Respiratory polygraphy was utilized on the BREAS SC-20 (Breas Clinical, Sweden) where the gadget, concentrated by a few specialists [19,20], measures the respiratory wind stream (through nasal cannula) also, stomach respiratory exertion (through piezoelectric groups). A gadget, APNiA (bti, Spain), with different channels, was concentrated by Dur an-Cantolla et al. [21]. It records the respiratory pattern (through a test coupled to a transducer), the oxygen immersion and heart recurrence (by beat oximetry), stomach exertion by inductive plethysmography, and snuffle sounds.

The NGP 140 (BITMED, Spain), concentrated by Candela et al. [22], moreover measures the respiratory exertion and a thermistor was utilized to distinguish the breathing pattern. The Edentrace II (Edentec, USA, otherwise called EMS 3711), was concentrated by _Alvarez et al. on the two grown-ups and kids [23,24] recording nasal wind current by thermistor, and chest developments utilizing a band. A chest-mounted gadget, Somnocheck (Weinman, Germany), was investigated by Oliveira et al. [25]. It records the airflow and snuffle by nasal cannula and $\mathrm{SpO} 2$ sensor. The Morpheus (Compumedics, Australia), concentrated by Takama furthermore, Kurabayashi [26], measures the wind current, utilizing a thermistor, $\mathrm{SpO} 2$ and respiratory developments with chest and stomach groups. ApneaGraph AG200 (MRA Medical, United Kingdom), concentrated by Singh et al. [27], inspects the cardio-respiratory wave utilizing a miniature weight and temperature transducer catheter to measure pressure and flow of air in pharynx.

- Review on other parameter acquisition

OSA are often detected by combining multiple approaches. Sound and pulse oximetry is used for detecting OSA is described in (Yadollahi and Moussavi [28]). Breathing sounds of trachea have been categorized after it has been recorded. Drop in spo2 signal will be analyzed with the help of a sound segment it is associated with. With the help of sigmoid function fuzzification is made and the obtained output is compared with a set threshold. Transportable Holter is used to record respiratory effort, ECG, oronasal airflow, and SpO2, is described in Dündar et al. [29]. In distributed time-delayed neural networks, standard deviation and average mean of HRV, oxygen desaturation and oronasal airflow were used as inputs for data classification. Heneghan et al. [31] also followed the same procedure in which he recorded ECG and $\mathrm{SpO} 2$ with the help of Holter device. The features obtained from the ECG-derived respiration, magnitude of QRS peaks, the $\mathrm{SpO} 2$ level, and heart rate variations are analyzed using the algorithm. The data classifications are done by LDA. SpO2 


\section{International Journal of Advanced Research in Computer and Communication Engineering}

Vol. 9, Issue 12, December 2020

DOI 10.17148/IJARCCE.2020.91204

and breathing sounds are observed with the help of Neck-cuff embedded sensors as described in Rofouei et al. [32]. To find periods of non-breathing and drops in the $\mathrm{SpO} 2$ level is sent analysis module via Bluetooth. Attaching a microphone to the patient's throat enables us to measure the patient's respiratory Al-Mardini et al. [33]. The Data collection and AHI determination is done with the help of smartphones. In order to determine Oxygen Desaturation Index (ODI), mobile phones are connected with a pulse oximeter. To determine the OSA occurrence an algorithm is used in which the mean of AHI and ODI obtained and compared with the threshold. SleepAp-The cell phone application, SleepAp, was introduced by Behar et al. [34] utilizing highlights gotten by sound recorded from an outer receiver, put close to the nose, and PPG recorded by a heartbeat oximeter associated with the application by Bluetooth, as contributions of a SVM classifier. On observation with the existing study, there is no research evidence for monitoring abrupt awakening pattern in targeted subjects. The proposed solution will be incorporated with accelerometer sensor (MPU6050) for monitoring abrupt awakening pattern. So far available devices ( $\mathrm{SpO} 2$ sensor, respiratory sensor, market available devices and other parameter acquisition devices) are been discussed. The drawbacks and additional addons of the proposed system will be discussed in discussions.

\section{DISSUSSIONS}

Despite of the advanced output there are some parameters which are unnoticed and not analyzed during continuous monitoring of sleep apnea. The drawback of the existing study in $\mathrm{SpO} 2$ sensor is that the data cannot be shared widely. But in proposed solution pulse oximeter sensor (MAX30100) will be integrated with IoT for wide range of access at low cost. In future GSM feature will also be studied and analyzed for transmission [30]. For respiration sensor additional add on feature incorporated is the automated process of supportive system which will be initiated when patient faces trouble in breathing. As per review on market available products which are discussed there are certain drawbacks such as in, Apnoescreen-I (CNS-Jaeger, Germany) [17], it is minimally invasive and cause discomfort to the patients. In Nigro et al. [18] wrist-mounted gadget, Wrist Ox 3100 (Nonin, USA) is used which is high cost and only $\mathrm{SpO} 2$ parameter is monitored. In Respiratory polygraphy [19-20] it is invasive and comparatively high cost. While in Candela et al. [22], there are no specific parameter to monitor. As per Alvarez et al. [23-24], nasal airflow is determined only by using thermistor. APNiA gadget which is concentrated by Dur an-Cantolla et al. [21] is minimally invasive and causes discomfort. As per the researchers [[25], [26], [27],] comparatively minimum parameters are measured and not integrated with IoT. Overall, there are no research evidence for monitoring abrupt awakening pattern in geriatric population. The proposed solution will be incorporated with accelerometer sensor (MPU6050) for monitoring abrupt awakening pattern. Moreover, the data transmission is also not yet discussed in any research, so the proposed solution will be given with encrypted with IoT feature.

\section{CONCLUSION}

In this review, the existing methodologies under $\mathrm{SpO} 2$, respiration sensor, commercial sensor and other miscellaneous sensor were studied and analyzed. The drawback of each type and its corresponding add-on feature was examined in systemic manner. The future work of this paper is to implement the proposed technology in real time manner, the experimentation and data acquition will be implemented and transferred to the concern physician through IoT for observation.

\section{REFERENCE}

1) "Sleep Apnea: What Is Sleep Apnea?". NHLBI: Health Information for the Public. U.S. Department of Health and Human Services. July 10, 2012. Archived from the original on August 19, 2016. Retrieved 2016-08-18.

2) Global Surveillance, Prevention and Control of Chronic Respiratory Diseases: A Comprehensive Approach. World Health Organization. 2007. p. 32. ISBN 9789241563468.

3) Benjafield AV, Ayas NT, Eastwood PR, Heinzer R, Ip MSM, Morrell MJ, Nunez CM, Patel SR, Penzel T, Pepin JL et al. Estimation of the global prevalence and burden of obstructive sleep apnoea: a literature-based analysis. Lancet Respir. Med. 2019; 7: 687-98.

4) Courtesy: $\quad$ Lung $\quad 2013$

5) Oliver N, Flores-Mangas F. HealthGear: automatic sleep apnea detection and monitoring with a mobile phone. J Commun 2007;2(2):1-9.

6) Burgos A, Goni A, Illarramendi A, Bermúdez J. Real-time detection of apneas on a PDA. IEEE Trans Inf Technol Biomed 2010;14(4):995-1002.

7) Zhang J, Zhang Q, Wang Y, Qiu C. A real-time auto-adjustable smart pillow system for sleep apnea detection and treatment. In: 2013 ACM/IEEE international conference on information processing in sensor networks; April, 2013.

8) Garde A, Dehkordi P, Wensley D, Ansermino J, Dumont G. Pulse oximetry recorded from the phone oximeter for detection of obstructive sleep apnea events with and without oxygen desaturation in children. In: 201537 th annual international conference of the IEEE engineering in medicine and biology society; August, 2015. 


\section{International Journal of Advanced Research in Computer and Communication Engineering}

Vol. 9, Issue 12, December 2020

DOI 10.17148/IJARCCE.2020.91204

9) Angius G, Raffo L. A sleep apnoea keeper in a wearable device for Continuous detection and screening during daily life. In: 2008 computers in cardiology; September, 2008.

10) Kaniusas E, Mehnen L, Pfützner H, Saletu B, Popovic R. Evaluation of acoustic signals for the detection of sleep apnea events. Proc Int Measur Confederation 2002; 7:177-82

11) Nakano H, Hayashi M, Ohshima E, Nishikata N, Shinohara T. Validation of a new system of tracheal sound analysis for the diagnosis of sleep apneahypopnea syndrome. Sleep 2004;27(5):951-7.

12) Zhao Y, Zhang H, Liu W, Ding S. A snoring detector for OSAHS based on patient's individual personality. In: 2011 3rd international conference on awareness science and technology; September, 2011

13) Lu-Ping F, Ze-Min M, Sheng-Sheng F. R\&D for home sleep apnea syndrome observation system. In: 2013 international conference on computer sciences and applications; December, 2013.

14) Ren Y, Wang C, Yang J, Chen Y. Fine-grained sleep monitoring: hearing your breathing with smartphones. In: 2015 IEEE conference on computer communications; April, 2015.

15) Han T, Min S, Nam Y. A real-time sleep monitoring system with a smartphone. In: 2015 9th international conference on innovative mobile and internet services in ubiquitous computing; July, 2015.

16) Nandakumar R, Gollakota S, Watson N. Contactless sleep apnea detection on smartphones. In: 13th annual international conference on mobile systems, applications, and services; May, 2015.

17) Golpe R, Jimenez A, Carpizo R. Home sleep studies in the assessment of sleep apnea/hypopnea syndrome. Chest 2002;122(4):1156-61.

18) Nigro C, Aimaretti S, Gonzalez S, Rhodius E. Validation of the WristOx $3100^{\mathrm{TM}}$ oximeter for the diagnosis of sleep apnea/hypopnea syndrome.Sleep Breath 2009;13(2):127-36.

19) Núnez R, Castro J, Socarras E, Calleja J, Rubio R, Aizpuru F, et al. Estudio de la validez de un equipo de poligrafía respiratoria (BREAS SC-20) para el diagnostico del syndrome de apneas-hipopneas durante el sueno. Archivos de Bronconeumología 2003;39(12):537-43.

20) Masa J, Corral J, Pereira R, Duran-Cantolla J, Cabello M, Hernandez-Blasco L, et al. Effectiveness of sequential automatic-manual home respiratory polygraphy scoring. Eur Respir J 2013;41(4):879-87.

21) Duran-Cantolla J, Almeida G, Guerenu V, Rotaeche L, Alkhraisat M, Carro J,et al. Validation of a new domiciliary diagnosis device for automatic diagnosis of patients with clinical suspicion of OSA. Respirology 2017;22(2):378-85.

22) Candela A, Hernandez L, Asensio S, Sanchez-Paya J, Vila J, Benito N, et al. Validation of a respiratory polygraphy system in the diagnosis of sleep apnea syndrome. Archivos de Bronconeumología 2005;41(2):71-7.

23) Alvarez M, Santos J, Guevara J, Martínez M, Pascual L, Banuelos J, et al. Fiabilidad de la poligrafía respiratoria domiciliaria para el diagnostico del síndrome de apneas-hipopneas durante el sueno. Analisis de costes. Archivos de Bronconeumología 2008;44(1):22-8.

24) Alvarez M, Santos J, Guevara J, Egüia A, Carbajo E, Jimenez J, et al. Fiabilidad de la poligrafía respiratoria para el diagno stico del síndrome de apneas-hipopneas durante el sueno en ninos. Archivos de Bronconeumología 2008;44(6):318-23.

25) Oliveira A, Martinez D, Vasconcelos L, Gonçalves S, Lenz M, Fuchs S, et al. Diagnosis of obstructive sleep apnea syndrome and its outcomes with home portable monitoring. Chest 2009;135(2):330-6.

26) Takama N, Kurabayashi M. Effectiveness of a portable device and the need for treatment of mild-to-moderate obstructive sleep-disordered breathing in patients with cardiovascular disease. J Cardiol 2010;56(1):73-8.

27) Singh A, Al-Reefy H, Hewitt R, Kotecha B. Evaluation of ApneaGraph in the diagnosis of sleep-related breathing disorders. Eur Arch Oto-RhinoLaryngol 2008;265(12):1489-94.

28) Yadollahi A, Moussavi Z. Acoustic obstructive sleep apnea detection. In:2009 annual international conference of the IEEE engineering in medicine and biology society; September, 2009.

29) Dündar T, Yılmaz A, Çaglar €O. Sleep apnea detection for prephase diagnosis using third level holter recording device. In: 2011 IEEE 19 th signal processing and communications applications conference; April, 2011.

30) Nithyaa A.N, Premkumar.R, Geethaaanandhi C., Gokul M., Manjusha.M. DIABETIC FOOT ULCERATION SCREENING VIA GSM SETUP ALONG WITH FLEXIFORCE SENSORS. JCR. 2020; 7(19): 6071-6077. doi:10.31838/jcr.07.19.702

31) Heneghan C, Chua C, Garvey J, Chazal P, Shouldice R, Boyle P, et al. A portable automated assessment tool for sleep apnea using a combined holter-oximeter. Sleep 2008;31(10):1432-9.

32) Rofouei M, Sinclair M, Bittner R, Blank T, Heffron J. A non-invasive wearable neck-cuff system for real-time sleep monitoring. In: 2011 international conference on body sensor networks; May, 2011.

33) Al-Mardini M, Aloul F, Sagahyroon A, Al-Husseini L. On the use of smartphones for detecting obstructive sleep apnea. In: 2013 IEEE $13^{\text {th }}$ international conference on bioinformatics and bioengineering; November, 2013.

34) Behar J, Roebuck A, Shahid M, Daly J, Hallack A, Palmius N, et al. SleepAp: an automated obstructive sleep apnoea screening application for smartphones. IEEE J Biomed Health Inform 2015;19(1):325-31. 